\title{
SÉANCE DU 3 AOUT 1922
}

PRÉSIDENGE DE M. PONS.

La séance est ouverte à 10 heures dans la grande salle de l'Hôtel de Ville d'Aiguilles-en-Queyras, mise gracieusement à la disposition de la Société par la Municipalité.

M. le Président prie M. Boursier, maire d'Aiguilles, de prendre place au Bureau.

Lecture est donnée par M. le Secrétaire du procès-verbal de la précédente séance, dont la rédaction est adoptée.

M. le Président a le regret d'annoncer le décès de M. Robert Mirande, Secrétaire de la Société et préparateur au Muséum d'Histoire naturelle de Paris, décédé des suites d'une douloureuse maladie contractée en captivité.

Il annonce ensuite les présentations suivantes :

MM. Vuathier (Charles), docteur en pharmacie, rue de l'Inquisition, 5, à Toulouse (HauteGaronne), présenté par MM. Comère et Gerber.

Pí́dallu (André), pharmacien-major de $1^{\text {re }}$ classe, rue des Géridaux, 11, à Sèvres (Seineet-0ise), présenté par MM. Réaubourg et Lutz.

En raison des usages adoptés par les Sessions et aucune opposition n'étant faite à ces canditatures, M. le Président proclame séance tenante MM. Vuathier et Piédallu membres de la Société.

La parole est ensuite donnée à M. le Maire d'Aiguilles qui remercie la Société d'avoir visité le Queyras et d'avoir choisi Aiguilles comme lieu de sa réunion de clòture.

Il présente ensuite un très ancien herbier local, conservé 
au Musée de la Ville et dont les membres présents prendront connaissance à l'issue de la séance.

Les communications suivantes sont alors développées par leurs auteurs.

\section{Note sur les zones de végétation fongique dans les Alpes}

PAR M. ROGER HEIM.

Jusqu'à ces derniers temps, l'influence possible de l'altitude sur la répartition, la morphologie ou l'anatomie des Champignons supérieurs n’avait pas été indiquée nettement avant que M. J. Costantin ait signalé, dans une récente communication à l'Académie des Sciences ${ }^{1}$, à la suite d'observations faites en septembre 1921 dans les Alpes de la Vanoise, que, selon lui, le facies montagnard donnait à certaines espèces délicates des particularités morphologiques spéciales caractérisées par une tendance au nanisme.

La lecture de cette communication nous a causé d'autant plus de plaisir qu'elle confirmait certaines observations que nous avions faites en septembre 1920 et en 1921 dans la HauteSavoie, et dont nous avions réservé la publication à plus tard.

Nous avons eu l'occasion durant la présente Session de la Société botanique dâns le Briançonnais ${ }^{2}$ et malgré la longue et extraordinaire période de sécheresse qui l'a précédée, de vérifier à nouveau et de compléter les remarques précédemment faites. C'est pourquoi nous croyons bon de donner ici un rapide aperçu de nos observations.

\section{$\stackrel{* *}{* *}$}

Dans les montagnes, les différents facteurs cosmiques agissent sur les Champignons probablement aussi intensivement que

1. Le 3 octobre 1921.

2. Du 23 juillet au 3 août 1922 . 


\section{$2 \mathrm{BHL}$ Biodiversity Heritage Library}

1922. "Séance Du 3 Aout 1922." Bulletin de la Société botanique de France 69, 463-464. https://doi.org/10.1080/00378941.1922.10833462.

View This Item Online: https://www.biodiversitylibrary.org/item/93160

DOI: https://doi.org/10.1080/00378941.1922.10833462

Permalink: https://www.biodiversitylibrary.org/partpdf/161277

\section{Holding Institution}

Missouri Botanical Garden, Peter H. Raven Library

\section{Sponsored by}

Missouri Botanical Garden

\section{Copyright \& Reuse}

Copyright Status: Public domain. The BHL considers that this work is no longer under copyright protection.

This document was created from content at the Biodiversity Heritage Library, the world's largest open access digital library for biodiversity literature and archives. Visit BHL at https://www.biodiversitylibrary.org. 